\title{
Noncommutative Geometry and Dynamical Models on $U(u(2))$ Background
}

Gurevich $\mathbf{D}^{1 *}$ and Saponov $\mathbf{P}^{2}$

${ }^{1}$ LAMAV, Universit de Valenciennes, France

${ }^{2}$ National Research University Higher School of Economics, Russia

\begin{abstract}
In our previous publications we have introduced a differential calculus on the algebra $U(\mathrm{gl}(\mathrm{m}))$ based on a new form of the Leibniz rule which differs from that usually employed in Noncommutative Geometry. This differential calculus includes partial derivatives in generators of the algebra $U(g l(m))$ and their differentials. The corresponding differential algebra $\Omega(U(g l(m)))$ is a deformation of the commutative algebra $\Omega(\operatorname{Sym}(g l(m)))$. A similar claim is valid for the Weyl algebra $W(U(g l(m)))$ generated by the algebra $U(g l(m))$ and the mentioned partial derivatives. In the particular case $m=2$ we treat the compact form $U(u(2))$ of this algebra as a quantization of the Minkowski space algebra. Below, we consider non-commutative versions of the Klein-Gordon equation and the Schrodinger equation for the hydrogen atom. To this end we de ne an extension of the algebra $U(u(2))$ by adding to it meromorphic functions in the so-called quantum radius and quantum time. For the quantum Klein-Gordon model we get (under an assumption on momenta) an analog of the plane wave, for the quantum hydrogen atom model we find the first order corrections to the ground state energy and the wave function.
\end{abstract}

Keywords: Weyl algebra; Leibniz rule; Quantum radius; Plane wave; Klein-Gordon model; Schrodinger model; Hydrogen atom model

AMS Mathematics Subject Classification, 2010: 46L65, 46L87, 81T75

\section{Introduction}

One of the basic problems of the Noncommutative (NC) Geometry is to construct a differential calculus on a given NC algebra $\boldsymbol{A}$. This calculus should involve the differential algebra $\Omega(\boldsymbol{A})$, generated by $\boldsymbol{A}$ and differentials $d a, a \in A$, and vector fields (in appropriate cases, analogs of partial derivatives). The core of the problem is a proper definition of the Leibniz rule for these vector fields and the corresponding de Rham operator. Especially, this problem is interesting for algebras arising from quantization of commutative ones since in this case the question of deformation properties of the algebra $\Omega(\boldsymbol{A})$ can be addressed.

The most common approach to this problem is based on the socalled Kahler forms which generate the universal differential algebra. In this algebra the usual Leibniz rule $d(a b)=d a b+a d b$ is preserved but the commutativity relation $a d b=d b a$ is canceled. As a result, the differential algebra becomes bigger than the usual one, even if $A$ is a commutative algebra, say the coordinate algebra of a regular algebraic variety.

Sometimes, the Hochschild homology $H_{i}(A)$ is considered as a convenient analog of the space $\Omega^{i}(A)$ (for the relations between these two objects for NC algebras $\boldsymbol{A}$ we refer the reader to Loday [1]. Whereas the Hochschild cohomology $H^{i}(A)$ plays the role of the space of polyvector fields on $\boldsymbol{A}$ (this is motivated by the famous HochschildKostant-Rosenberg theorem). In particular, the first group $H^{1}(A)$ is identified with the space of derivations (quotiented over the inner ones), i.e. operators subject to the usual Leibniz rule. An advanced calculus based on this treatment of the Hochschild (co)homology can be found in the papers [2-4] and others [1,5]. Nevertheless, if $\boldsymbol{A}$ is a coordinate algebra of a regular variety, the space $H_{i}(A)$ differs from that $\Omega^{i}(A)$ considered in its classical sense.

The same is valid if we replace $H_{i}(A)$ here by the cyclic homology $H C_{i}(A)$ due to Connes. He also introduced another complex in which the role of the de Rham operator is played by the commutator with a
Dirac operator. However, it differs from the usual de Rham complex as well.

Also, observe that in all mentioned approaches no analogs of partial derivatives are introduced, even if a given NC algebra is a deformation of the coordinate algebra of a linear space.

A couple of years ago we suggested a new type of differential calculus [6,7] on the algebras $U(g l(m))$, the super-algebras $U(g l(m \mid n))$, and their braided analogs the so-called modified Reflection Equation algebra $L(R)$ related to a Hecke symmetry $R$. The calculus is based on so-called permutation relations between elements of the NC algebra in question and partial derivatives on it.

If such a Hecke symmetry $R$ is a deformation of the usual flip $P$, the corresponding Reflection Equation algebra $L(R)$ admits a construction of the differential algebra which is a deformation of its classical counterpart. Moreover, the corresponding Weyl algebra, generated by $L(R)$ and the mentioned partial derivatives, is also a deformation of its classical counterpart. Besides, if $R$ is involutive $\left(R^{2}=I\right)$ the mentioned permutation relations can be expressed via a new form of the Leibniz rule which in its turn is related to a coproduct in the algebra generated by the partial derivatives.

Below we assume $R$ to be the usual flip $P$. Moreover, we deal with a particular case $m=2$, i.e. our basic NC algebra is $\left.U(g l(2))_{h}\right)$ or rather its compact counterpart $U\left(u(2)_{h}\right)$. The latter algebra can be treated as a NC (quantum) analog of the Minkowski space algebra. Emphasize that the algebra $U\left(u(2)_{h}\right)$ is a deformation (quantization) of the commutative

*Corresponding author: Gurevich D, LAMAV, Universit de Valenciennes, 59313 Valenciennes, France, Tel: +33 (0)3 2751 12 34; E-mail: gurevich@univ-valenciennes.fr Received January 17, 2015; Accepted June 16, 2015; Published June 18, 2015

Citation: Gurevich D, Saponov P (2015) Noncommutative Geometry and Dynamical Models on U(u(2)) Background. J Generalized Lie Theory Appl 9: 215 doi:10.4172/1736-4337.1000215

Copyright: () 2015 Gurevich D, et al. This is an open-access article distributed under the terms of the Creative Commons Attribution License, which permits unrestricted use, distribution, and reproduction in any medium, provided the original author and source are credited. 
algebra $\operatorname{Sym}(u(2))$ which is considered as the Minkowski space algebra provided it is endowed with a proper metric.

In this connection we want to mention other deformations of the Minkowski space algebra such as q-Minkowski space algebra (in fact it is a particular case of the Reflection Equation algebra). There is a large number of publications devoted to these NC versions of the Minkowski space algebra.

Note, that our version of the quantum Minkowski space algebra, namely $U\left(u(2)_{h}\right)$ has a lot of advantages with respect to the other ones.

First of all, the algebra $U\left(u(2)_{h}\right)$ is an $S O(3)$-covariant enveloping algebra of the reductive algebra $u(2)_{h}$ whose simple component is the Lie algebra $s u(2)_{h}$. In virtue of the Kostant's theorem (see section 2) the algebra $U\left(s u(2)_{h}\right)$ admits a decomposition in the direct sum of isotypic components.

$$
\bigoplus_{k=0}\left(Z\left(U\left(s u(2)_{h}\right)\right) \otimes V^{k}\right)
$$

where $Z\left(U\left(s u(2)_{h}\right)\right)$ is the center of the algebra $U\left(s u(2)_{h}\right)$ and $V^{k} \subset$ $U\left(s u(2)_{h}\right)$ is the irreducible $S U(2)$-module $\left(\operatorname{dim} V^{k}=2 k+1\right)$. This decomposition provides us with a basis in the algebra $U\left(s u(2)_{h}\right)$ convenient for our computations.

Besides, in the algebra $U\left(u(2)_{h}\right)$ there is a version of the CayleyHamilton $(\mathrm{CH})$ identity for a matrix $N$ composed of the $U\left(u(2)_{h}\right)$ generators. The roots $\mu_{1}$ and $\mu_{2}$ of this $\mathrm{CH}$ identity ("eigenvalues" of the matrix $N$ ) are related to a quantity which can be treated as a quantum analog of the radius $\sqrt{x^{2}+y^{2}+z^{2}}$. This "quantum radius" $r_{h}=\frac{1}{2 i}\left(\mu_{1}-\mu_{2}\right)$ together with the quantity $\tau=\frac{\mu_{1}+\mu_{2}-h}{2 i}$ (treated as a quantum analog of the time) is very useful for a parametrization of the center $Z\left(U\left(\operatorname{su}(2)_{h}\right)\right)$. It also enables us to construct a central extension of the algebra $U\left(s u(2)_{h}\right)$ (and consequently, of $\left.U\left(u(2)_{h}\right)\right)$ needed for constructing NC versions of certain physical models.

In the present paper we are dealing with the model of the free massive scalar field described by the Klein-Gordon equation and with the model of a particle in a central potential field (the hydrogen atom) described by the Schrodinger equation. In our previous publication we have considered the Klein-Gordon equation in nontrivial rotationally invariant metric. Below we study the simplest form of the NC KleinGordon equation and find its plane wave type solution. For this solution we exhibit (under an assumption on momenta) a quantum deformation of the so-called relativistic dispersion relation $E^{2}=m^{2} c^{4}$ $+p^{2} c^{2}$ connecting the energy $E$ of a particle of the mass $m$ and the square of its momentum $p^{2}$. As for the NC Schrodinger equation, we find the first order corrections to the ground state energy and to the wave function of the corresponding hydrogen atom model.

Emphasize that there are known numerous attempts to generalize some models of mathematical physics to quantum algebras. Besides the aforementioned versions of the Minkowski space algebra, we want to cite the papers $[8,9]$ where some rotationally invariant models were quantized. The essential dissimilarity between the cited papers and ours consists in different treatment of the differential operators (such as the Laplacian $\Delta=\partial_{\mathrm{x}}^{2}+\partial_{\mathrm{y}}^{2}+\partial_{\mathrm{y}}^{2}$ ) appearing in dynamical equations of the models. In the cited papers the partial derivatives are treated in the classical sense, only the usual commutative product of functions is replaced by its deformed version (called $\star$-product). By contrary, in our approach the partial derivatives are directly defined on the $\mathrm{NC}$ algebra in question and their properties differ drastically from those of the usual derivatives.
First, the partial derivatives in the spacial generators $x, y, z$ (and consequently, the Laplacian) act nontrivially on the time variable and vice versa.

Second, we treat the quantized models in terms of the quantum algebra without using any $\star$-product. The only problem arising in our approach is pulling an operator which describes the model forward to the quantum algebra. We call this procedure the quantization of operators. For the operators we are dealing with, this quantization is easy: the derivatives should be treated in the new sense (i.e. according to our definition of the derivatives on the algebra $U(u(2)))$ ) and the radial variable $r$ in a potential $V(r)$ should be replaced by its quantum counterpart $r_{\hbar}$, as well as the time variable $t$ is replaced by its quantum counterpart $\tau$. In more detail the problem of quantization of differential operators is considered in Gurevich et al. [7].

Third, the models in question can be restricted to any component coming in the sum (1.1) and then to some lattices in 2-dimensional space "time-quantum radius". This property is due to the fact that the partial derivatives in time and quantum radius are difference operators. In this sense we speak about a discretization of the space-time.

The ground field $\mathbb{K}$ is assumed to be $\mathbb{R}$ or $\mathbb{C}$, its concrete form is always clear from the context.

The paper is organized as follows. In the next section we introduce a quantum version of the Weyl algebra. Also, we define its extended version by adding meromorphic functions in the quantum radius and the time to it. In the section 3 we give an operator meaning to elements of the quantum Weyl algebra they become operators acting on the algebra $U\left(u(2)_{h}\right)$. In section 4 we introduce the partial derivative in quantum radius and clarify its role in discretization of models we are dealing with. Section 5 is devoted to our quantization of the aforementioned rotationally invariant dynamical models. In section 6 we present some concluding remarks and discuss the perspective of our study. In Appendix we get together some technical results used in the text.

\section{The Weyl Algebra $W\left(U\left(u(2)_{h}\right)\right)$ and its Extension}

First, recall that according to Gurevich D et al., [6,7] the Weyl algebra $W\left(U\left(g l(m)_{h}\right)\right)$ is a unital associative algebra generated by $2 m^{2}$ elements $\left\{n_{i}^{j}\right\}_{1 \leq i, j \leq m}$ and $\left\{d_{i}^{j}\right\}_{1 \leq i, j \leq m}$. Having defined the $m \times m$ generating matrices $N=\backslash n_{i}^{j} \backslash$ and $D=\backslash d_{i}^{j} \backslash$, we present the multiplication rules of the $W\left(U\left(g l(m)_{h}\right)\right)$ generators in the following matrix form:

$$
\begin{aligned}
& P N_{1} P N_{1}-N_{1} P N_{1} P=h\left(P N_{1} N_{1} P\right), \\
& P D_{1} P D_{1}=D_{1} P D_{1} P, \\
& D_{1} P N_{1} P-P N_{1} P D_{1}=P+h D_{1} P
\end{aligned}
$$

Where $h$ is a numeric parameter, $N_{1}=N \otimes I_{m \times m}, \mathrm{D}_{1}=D \otimes I_{m \times m} a n d m^{2} \times m^{2}$ matrix $P$ is the usual flip.

As is clear from the first line of the system $(2.1), n_{i}^{j}$ are generators of the sub algebra $U\left(g l(m)_{h}\right) \subset W\left(U\left(g l(m)_{h}\right)\right)$. The second line expresses the commutativity of generators $d^{j}{ }_{i}$. The corresponding Abelian subalgebra will be denoted $D$. The relations coming in the third line are called the permutation relations. The permutation relations allow us to treat the generators $\left\{\mathrm{d}^{j}{ }_{i}\right\}$ as (quantum) partial derivatives. In particular, in the classical limit $(h=0)$ the element $d_{i}$ can be identified with the partial derivative $\partial / \partial n_{j}^{i}$. From now on we shall use the term "partial derivatives" for the elements $d_{i}^{j}$, while the precise operator meaning will be given to them in the next section. 
Note that the algebra $W\left(U\left(g l(m)_{h}\right)\right)$ can be treated as the enveloping algebra of a semidirect product of the Lie algebra $g l(m)_{h}$ with the basis $n_{i}^{j}$ and the commutative algebra $D=g l(m)_{0}$ with the basis $d_{i}^{j}$. In fact we have a slight modification of the semidirect product because of the constant terms, entering the flip $P$ in the third line of (2.1), but this does not impact the claim that in virtue of the PBW theorem the family of elements

$$
\left(n_{1}^{1}\right)^{a_{1}^{1}}\left(n_{1}^{2}\right)^{a_{1}^{2}} \ldots\left(n_{m}^{m}\right)^{a_{m}^{m}}\left(d_{1}^{1}\right)^{b_{1}^{1}}\left(d_{1}^{2}\right)^{b_{1}^{2}} \ldots\left(d_{m}^{m}\right)^{b_{m}^{m}}, \quad a_{i}^{j}, b_{i}^{j} \in \mathbb{N}
$$

is a basis of the algebra $W\left(U\left(g l(m)_{h}\right)\right)$.

Remark 1: Meljanac and Skoda have suggested the realization of relations (2.1) analogous to their construction from [MS] (also, see [S]). Let $\tilde{N}=\backslash t_{i}^{j} \backslash$ be the generating matrix of the algebra $\operatorname{Sym}(g l(m))$ (i.e. it satisfies the first line relations (2.1) with $\mathrm{h}=0$ ) and $\mathrm{D}=\backslash d_{i}^{j} \backslash$ be the matrix composed of the usual partial derivatives in $t_{i}^{j}: d_{i}^{j}=\partial / \partial t_{j}^{i}$. Then the matrices $N=\tilde{N}+h \tilde{N} D$ and $D$ satisfy the system (2.1).

Now, we write down the relations (2.1) in the particular case $m=2$ and pass to the compact counterpart $U\left(u(2)_{h}\right)$ of the algebra $U\left(g l(2)_{h}\right)$. To simplify formulae we use the following notation for the $U\left(g l(2)_{h}\right)$ generators:

$$
n_{1}^{1}=a, \quad n_{1}^{2}=b, \quad n_{2}^{1}=c, \quad n_{2}^{2}=d .
$$

The generators of the algebra $U\left(u(2)_{h}\right)$

$$
t=\frac{a+d}{2}, \quad x=\frac{i(b+c)}{2}, \quad y=\frac{c-b}{2}, \quad z=\frac{i(a-d)}{2} . \text { (2.2) abcd }
$$

are subject to the system of relations

$$
[x, y]=h z, \quad[y, z]=h x, \quad[z, x]=h y, \quad[t, x]=[t, y]=[t, z]=0 .
$$

The center $Z\left(U\left(u(2)_{h}\right)\right)$ is generated by the element $t$ and quadratic Casimir element

Cas $=x^{2}+y^{2}+z^{2}$.

The partial derivatives in $t, x, y$ and $z$ will be denoted as $\partial_{t}, \partial_{x}, \partial_{y}$ and $\partial_{z}$ respectively.

Besides, it is convenient to introduce a shifted derivative $\tilde{\partial}_{t}=\partial_{t}+\frac{2}{h} I$.

In terms of generators $t, x, y, z$ and the corresponding partial derivatives the third line of the system (2.1) reads

$$
\begin{array}{llll}
\tilde{\partial}_{t} t-t \tilde{\partial}_{t}=\frac{h}{2} \tilde{\partial}_{t} & \tilde{\partial}_{t} x-x \tilde{\partial}_{t}=-\frac{h}{2} \partial x & \tilde{\partial}_{t} y-y \tilde{\partial}_{t}=-\frac{h}{2} \partial y & \tilde{\partial}_{t} z-z \tilde{\partial}_{t}=-\frac{h}{2} \partial_{z} \\
\partial_{x} t-t \partial_{x}=\frac{h}{2} \partial_{x} & \partial_{x} x-x \partial_{x}=\frac{h}{2} \tilde{\partial}_{t} & \partial_{x} y-y \partial_{x}=\frac{h}{2} \partial z & \partial_{x} z-z \partial_{x}=-\frac{h}{2} \partial_{y} \\
\partial_{y} t-t \partial_{y}=\frac{h}{2} \partial_{y} & \partial_{y} x-x \partial_{y}=-\frac{h}{2} \partial_{z} & \partial_{y} y-y \partial_{y}=\frac{h}{2} \tilde{\partial}_{t} & \partial_{y} z-z \partial_{y}=\frac{h}{2} \partial_{x} \\
\partial_{z} t-t \partial_{z}=\frac{h}{2} \partial_{z} & \partial_{z} x-x \partial_{z}=\frac{h}{2} \partial_{y} & \partial_{z} y-y \partial_{z}=-\frac{h}{2} \partial_{x} & \partial_{z} z-z \partial_{z}=\frac{h}{2} \tilde{t}_{t}
\end{array}
$$

Our next aim is to introduce quantum analogs of "radial variables". To this end we need a version of the Cayley-Hamilton $(\mathrm{CH})$ identity for the generating matrix $N$

$$
N=\left(\begin{array}{ll}
a & b \\
c & d
\end{array}\right)\left(\begin{array}{ll}
t-i z & -i x-y \\
-i x+y & t+i z
\end{array}\right)
$$

In this case the corresponding characteristic polynomial $\chi(\lambda)$ is quadratic and the $\mathrm{CH}$ identity explicitly reads

$$
\mathrm{x}(N)=N^{2}-(2 t+h) N+\left(t^{2}+x^{2}+y^{2}+z^{2}+h t\right) I=0 .
$$

Note, that the coefficients of the above identity are central in the algebra $U\left(u(2)_{h}\right)$. Recall, that this is also true in the general case $U(g l(m) h)$.
The roots $\mu_{1}$ and $\mu_{2}$ of the characteristic polynomial are called the eigenvalues of the matrix $N$. This means that they solve the system of two algebraic equations

$$
\mu_{1}+\mu_{2}=2 t+h, \quad \mu_{1} \mu_{2}=t^{2}+x^{2}+y^{2}+z^{2}+h t,
$$

and, consequently, the eigenvalues should be treated as elements of an algebraic extension of the center $Z\left(U\left(u(2)_{h}\right)\right)$.

Equations (2.6) allow us to express the center generators (and, therefore, any element of $\left.Z\left(U\left(u(2)_{h}\right)\right)\right)$ in terms of $\mu_{1}$ and $\mu_{2}$ :

$$
t=\frac{\mu_{1}+\mu_{2}-h}{2}, \quad \mathrm{Cas}=\frac{h^{2}-\mu^{2}}{4} .
$$

(2.7) cas-mm

Hereafter, we use the notation $\mu=\mu_{1}-\mu_{2}$

Remark 2: Note that the quantity $\mu$ is defined up to a sign since the ordering of the eigenvalues is arbitrary.

Now, we compute the permutation relations of the partial derivatives $\partial_{x}, \partial_{y}, \partial_{z}$. and the Casimir element. In contrast with the commutative case, the other elements of the algebra $W\left(U\left(u(2)_{h}\right)\right)$ turn out to be involved in these permutation relations. We need the following operators

$$
Q=x \partial_{x}+y \partial_{y}+z \partial_{z}, \quad X=y \partial_{z}-z \partial_{y}, \quad Y=z \partial_{x}-x \partial_{z}, \quad Z=x \partial_{y}-y \partial_{x} .
$$

\section{Theorem 3:}

$$
\left(\begin{array}{c}
\partial_{x} \\
x \tilde{\partial}_{t} \\
x Q \\
X
\end{array}\right) \operatorname{cas}=\left(\begin{array}{cccc}
\operatorname{cas}-\frac{3}{4} h^{2} & h & 0 & h \\
0 & c a s-\frac{3}{4} h^{2} & -h & 0 \\
0 & h \text { Cas } & c a s+\frac{h^{2}}{4} & 0 \\
-h \text { Cas } & h^{2} & h & c a s+\frac{h^{2}}{4}
\end{array}\right)\left(\begin{array}{c}
\partial_{x} \\
x \tilde{\partial}_{t} \\
x Q \\
x
\end{array}\right) \text { (2.9) form }
$$

The permutation relation of $\partial_{y}$, (respectively, $\left.\partial_{z}\right)$ with the Casimir element are obtained from (2.9) by replacing $x$ and $X$ for $y$ and $Y$ (respectively, for $z$ and $Z$ ).

We denote the $4 \times 4$ matrix in formula (2.9) by $\Psi(\mathrm{Cas})$. Being expressed in terms of $\mu$ (see (2.7)) it reads

$$
\Psi(\mathrm{cas})=\left(\begin{array}{cccc}
\frac{-\mu^{2}-2 h^{2}}{4} & h & 0 & h \\
0 & \frac{-\mu^{2}-2 h^{2}}{4} & -h & 0 \\
0 & h \frac{h^{2}-\mu^{2}}{4} & \frac{2 h^{2}-\mu^{2}}{4} & 0 \\
-h \frac{h^{2}-\mu^{2}}{4} & h^{2} & h & \frac{2 h^{2}-\mu^{2}}{4}
\end{array}\right)
$$

The matrix $\Psi$ (Cas) is semisimple with the following spectrum:

$\Psi($ Cas $) \sim \operatorname{diag}\left(\lambda_{1}, \lambda_{1}, \lambda_{2}, \lambda_{2}\right), \quad \lambda_{1}=-\frac{\mu^{2}-2 h \mu}{4}, \quad \lambda_{2}=-\frac{\mu^{2}+2 h \mu}{4}$.

Its spectral decomposition reads

$$
\Psi(\mathrm{Cas})=\lambda_{1} P_{1}(\mu)+\lambda_{2} P_{2}(\mu), \quad P_{1}(\mu)=\frac{\Psi(\mathrm{Cas})-\lambda_{2} I}{\lambda_{1}-\lambda_{2}}, \quad P_{2}(\mu)=\frac{\Psi(\mathrm{Cas})-\lambda_{1} I}{\lambda_{2}-\lambda_{1}},
$$

where $I$ is the unit $4 \times 4$ matrix. The explicit matrix form of the complementary projectors $\mathrm{Pi}(\mu)$ is as follows: 


$$
P_{1}(\mu)=\frac{1}{\mu}\left(\begin{array}{cccc}
\frac{\mu-h}{2} & 1 & 0 & 1 \\
0 & \frac{\mu-h}{2} & -1 & 0 \\
0 & \frac{h^{2}-\mu^{2}}{4} & \frac{\mu+h}{2} & 0 \\
\frac{\mu^{2}-h^{2}}{4} & h & 1 & \frac{\mu+h}{2}
\end{array}\right), \quad P_{2}(\mu)=\frac{1}{\mu}\left(\begin{array}{cccc}
\frac{\mu+h}{2} & -1 & 0 & -1 \\
0 & \frac{\mu+h}{2} & 1 & 0 \\
0 & \frac{\mu^{2}-h^{2}}{4} & \frac{\mu-h}{2} & 0 \\
\frac{h^{2}-\mu^{2}}{4} & -h & -1 & \frac{\mu-h}{2}
\end{array}\right) \text {. }
$$

The permutation relations of the column entering (2.9) with the element Cas ${ }^{\mathrm{p}}, p \geq 2$, can be obtained in the same way but the matrix $\Psi$ (Cas) must be replaced by its $\mathrm{p}$-th degree. Using the spectral decomposition, we calculate $\Psi^{p}$ (Cas) as

$$
\Psi^{p}(\text { Cas })=\lambda_{1}^{p} P_{1}(\mu)+\lambda_{2}^{p} P_{2}(\mu) \text {. }
$$

So, we can find the permutation relations of the set of elements $\partial_{x}$, $x \tilde{\partial}_{t}, x Q$ and $X$ with any polynomial or even a series $f(u)$ in $u=$ Cas. The only thing we need for this purpose is

$$
f(\Psi(\text { Cas }))=f\left(\lambda_{1}\right) P_{1}(\mu)+f\left(\lambda_{2}\right) P_{2}(\mu) .
$$

However, we want to get the permutation relations of the above operators with $\mu$ and its powers $\mu^{p}, p \in \mathbb{Z}$. Since $\mu=f($ Cas) where $f(u)$ $=\sqrt{h^{2}-4 u}$, We get

$$
f\left(\lambda_{1}\right)=\sqrt{(\mu-h)^{2}}=\mu-h, f\left(\lambda_{2}\right)=\sqrt{(\mu+h)^{2}}=\mu+h .
$$

The sign is motivated by the classical limit. Finally, we arrive to the following permutation relations

$$
\left(\begin{array}{c}
\partial_{x} \\
x \tilde{\partial}_{t} \\
x Q \\
X
\end{array}\right) \mu^{p}=(\mu-h)^{p} P_{1}(\mu)\left(\begin{array}{c}
\partial_{x} \\
x \tilde{\partial}_{t} \\
x Q \\
X
\end{array}\right)+(\mu+h)^{p} P_{2}(\mu)\left(\begin{array}{c}
\partial_{x} \\
x \tilde{\partial}_{t} \\
x Q \\
X
\end{array}\right) \text {. (2.11) formm }
$$

In the same way the permutation relations of the partial derivatives $\partial_{y}$ and $\partial_{z}$ with $\mu^{p}, p \in \mathbb{Z}$ can be found. As for the permutation relations of the operators $\bar{\partial}_{t}$ and $Q$ with $\mu^{p}$, they can be extracted from formula (2.11): it suffices to cancel $x$ in the second and third lines of this matrix.

Thus, we arrive to the result (also, see [GS2] where these formulae were first obtained)

$$
\begin{aligned}
& \bar{\partial}_{t} \mu^{p}=\frac{(\mu+h)^{p}}{\mu}\left(Q+\frac{\mu+h}{2} \bar{\partial}_{t}\right)-\frac{(\mu-h)^{p}}{\mu}\left(Q-\frac{\mu-h}{2} \bar{\partial}_{t}\right) \\
& Q \mu^{p}=\frac{(\mu+h)^{p}}{\mu} \frac{(\mu-h)}{2}\left(Q+\frac{\mu+h}{2} \bar{\partial}_{t}\right)+\frac{(\mu-h)^{p}}{\mu} \frac{(\mu+h)}{2}\left(Q-\frac{\mu-h}{2} \bar{\delta}_{t}\right) .
\end{aligned}
$$

Note that in the classical case $(h=0)$ the variable $\mu$ is a multiple of the radius $r=\sqrt{x^{2}+y^{2}+z^{2}}$, namely, $\mu=2 i r$. In the general case $(h \neq 0)$ we introduce an analog of the radius by a similar formula.

Definition 4: The quantity $r_{\hbar}=\frac{\mu}{2 i}$ is called the quantum radius.

Below, we use the element $r_{\hbar}$ as well as $\mu$. However, dealing with $r_{\hbar}$ we prefer to use the renormalized deformation parameter $\hbar=\frac{h}{2 i}$ in order to avoid complex shifts of the type $r_{\hbar} \rightarrow r_{\hbar}+i a$ (as for the factor 2 it is introduced for the future convenience). For the same reason we will use the renormalized time $\tau=-i t$. All our formulae represented in terms of the initial quantities $(\mu, t, h)$ can be easily rewritten in terms of $\left(r_{\hbar}, \tau, \hbar\right)$ and vice versa. For example, the Casimir element in terms of new quantities has the form

$$
\text { Cas }=r_{\hbar}^{2}-\hbar^{2}
$$

We also define the partial derivative $\partial_{T}=i \partial_{t}$ and the corresponding "shifted derivative" $\bar{\partial}_{T}=i \bar{\partial}_{t}=\partial_{T}+\frac{1}{\hbar} I$. Note, that $\left[\partial_{\tau}, \tau\right]=\hbar \partial_{\tau}$.

Introduce now the commutative algebra $\mathbb{k}\left(\tau, r_{\hbar}\right)$ of functions in $\tau$ and $r_{\hbar} \mathrm{r}$ any $f\left(\tau, r_{\hbar}\right) \in\left(\tau, r_{\hbar}\right)$ we assume the function $f\left(z_{1}, z_{2}\right)$ to be meromorphic functions in two complex variables.

Next, consider the algebra

$A_{\hbar}=\left(U\left(\operatorname{su}(2)_{\mathrm{h}}\right) \otimes \mathbb{k}\left(\tau, \mathrm{r}_{\hbar}\right)\right) /\left\langle x^{2}+y^{2}+z^{2}-r_{h}^{2}+\hbar^{2}\right\rangle$.

The generators $\tau$ and $r_{\hbar}$ are assumed to commute with any element of the algebra $A_{\hbar}$ Thus, the subalgebra $\mathbb{k}\left(\tau, r_{\hbar}\right)$ forms evidently the center $Z\left(A_{\hbar}\right)$ of this algebra.

Our next aim is to find the permutation relations of the partial derivatives $\bar{\partial}_{\tau}, \ldots, \bar{\partial}_{z}$ and elements of the algebra $\mathbb{k}\left(\tau, r_{\hbar}\right)$.

First, consider in $\mathbb{k}\left(\tau, r_{\hbar}\right)$ polynomial functions in two variables. Since $\tau$ and $r_{\hbar}$, any polynomial $f\left(\tau, r_{\hbar}\right)$ can be presented as a finite sum $f\left(\tau, r_{\hbar}\right)=\sum g_{i}(\tau) q_{i}\left(r_{\hbar}\right)$ where $g_{i}$ and $q_{i}$ are some polynomials in one variable. Then, as was shown in [GS2], the permutation relations of the partial derivatives with a polynomial $g(\tau)$ are

$$
\bar{\partial}_{\tau} g(\tau)=g(\tau+\hbar) \bar{\partial}_{\tau}, \quad \partial_{a} g(\tau)=g(\tau+\hbar) \partial_{a}, \quad a \in\{x, y, z\} .
$$

$r^{p}$ As for the permutation relations of the partial derivatives with $r_{\hbar}^{p}$ (and, consequently, with any polynomial $f\left(r_{\hbar}\right)$ they can be easily deduced from (2.11).

Therefore, the above considerations together with (2.14) and (2.11) allow us to get for any polynomial $f\left(\tau, r_{\hbar}\right)$ in two variables the following result

$$
\partial_{x} f\left(\tau, \mathrm{r}_{h}\right)=\frac{f\left(\tau+\hbar, \mathrm{r}_{\mathrm{h}}+\hbar\right)}{2 r_{h}}\left(\left(\mathrm{r}_{\mathrm{n}}+\hbar\right) \partial_{x}+x \tilde{\partial}_{\tau}+i X\right)+\frac{f\left(\tau+\hbar, \mathrm{r}_{h}-\hbar\right)}{2 r_{h}}\left(\left(\mathrm{r}_{h}-\hbar\right) \partial_{x}-x \tilde{\partial}_{\tau}-i X\right),
$$

and similarly for the derivatives $\partial_{y}$ and $\partial_{z}$.

For the partial derivative $\bar{\partial}_{\tau}$ we find

$$
\bar{\partial}_{\tau} f\left(\tau, r_{h}\right)=\frac{f\left(\tau+\hbar, \mathrm{r}_{h}+\hbar\right)}{2 r_{h}}\left(\left(\mathrm{r}_{h}+\hbar\right) \bar{\partial}_{\tau}+Q\right)+\frac{f\left(\tau+\hbar, \mathrm{r}_{h}-\hbar\right)}{2 r_{h}}\left(\left(\mathrm{r}_{h}-\hbar\right) \bar{\partial}_{\tau}-Q\right) .
$$

We extend these formulae from polynomials to arbitrary functions $f\left(\tau, \mathbf{r}_{h}\right) \in \mathbb{k}\left(\tau, \mathbf{r}_{h}\right)$ by definition.

Now, define the algebra $W\left(A_{\hbar}\right)$ to be the algebra generated by and the partial derivatives $\bar{\partial}_{\tau}, \ldots, \bar{\partial}_{z}$ endowed with the permutation relations described by formulae (2.3), (2.15) with its $y$ - and $z$-analogs and (2.16). We call $W\left(A_{\hbar}\right)$ the extended Weyl algebra.

Below, we define the derivative $\partial_{r h}$ in $W\left(A_{\hbar}\right)$ but it will be expressed in terms of already existing elements of $W\left(A_{\hbar}\right)$ so we shall not need its new extension.

By resuming this section, we want to emphasize that the algebra $W($ $A_{h}$ is introduced as a quotient-algebra. Intuitively, it is clear that it is a deformation of its classical counterpart, de fined by formula (2.13) with $h=0$. However, the fact that the algebra $W\left(U\left(u(2)_{h}\right)\right)$ is a deformation of its classical counterpart $W(\operatorname{Sym}(u(2)))$ can be shown rigorously. Indeed, it follows from the discussed above treatment of the algebra $W\left(U\left(g l(m)_{h}\right)\right)$ as the enveloping algebra of a semidirect product.

\section{Differential Operators on the Algebra $A_{\hbar}$}

Now, we are going to define an action of the algebra $W\left(A_{\hbar}\right)$ onto $A_{\hbar}$. To this end we first define this action for the "partial derivatives" $d_{i}\left(\right.$ recall that at the limit $h=0$ we have $d_{i}^{j}=\partial_{n_{j}^{j}}$ )

$$
D \otimes U\left(g l(m)_{\mathrm{h}}\right) \rightarrow U\left(g l(m)_{h}\right): \quad d_{i}^{j} \otimes n \mapsto \bar{n}=d_{i}^{j}(n) .
$$


Hereafter, the action of an operator $D$ on an element $n € U\left(u(m)_{h}\right)$ will be denoted $D(n)$, while we keep the notation $D n=\bar{n} \bar{D}$ for the permutation relation.

Below we need the counit map $\varepsilon: \mathrm{D} \rightarrow \mathbb{k}$ introduced by

$\varepsilon\left(1_{D}\right)=1, \quad \varepsilon\left(d_{i}^{j}\right)=0, \quad \varepsilon\left(d_{1} d_{2}\right)=\varepsilon\left(d_{1}\right) \varepsilon\left(d_{2}\right), \quad \forall d_{1}, d_{2} \in D . \quad$ (3.1) coun

The symbol $1_{A}$ stands for the unit element of the (sub) algebra $A$.

Then we proceed as follows. Given a product of elements $d n$ (here after, we omit the sign $\otimes$ ), we first apply the permutation relations (the third line of (2.1)) to the elements $d$ and $n$ in order to move elements of $D$ to the most right position

$$
d n=\sum_{i} n_{i}^{\prime} d_{i}^{\prime}+n^{\prime \prime} 1_{D}, \quad n_{i}^{\prime}, n^{\prime \prime} \in U\left(g l(m)_{h}\right), \quad d_{i}^{\prime} \in D,
$$

where in the right hand side we have a finite sum of elements. After that, we apply the counit map $\varepsilon$ to all elements of $D$ in the above sum and get an element from $U\left(g l(m)_{h}\right)$ :

$$
\sum_{i} n_{i}^{\prime} d_{i}^{\prime}+n^{\prime \prime} 1_{D} \stackrel{\mathrm{id} \otimes \varepsilon}{\rightarrow} \sum_{i} n_{i}^{\prime} \varepsilon\left(d_{i}^{\prime}\right)+n^{\prime \prime} \varepsilon\left(1_{D}\right)=n^{\prime \prime},
$$

where we identify $U\left(g l(m)_{h}\right) \otimes$ with $U\left(g l(m)_{h}\right)$.

Thus, all partial derivatives $\partial_{t}, \ldots, \partial_{z}$ become operators acting on $U(u(2))_{1}$. Emphasize that the action of the partial derivatives is not subject to the classical Leibniz rule. By contrary, these derivatives meet a new version of the Leibniz rule which was found by Meljanac and Skoda [10] (see also [6] where we have given another but equivalent form of this Leibniz rule). In order to describe this form of the Leibniz rule, consider the following coproduct in the algebra $D$

$$
\Delta\left(d_{i}^{j}\right)=d_{i}^{j} \otimes 1+1 \otimes d_{i}^{j}+h \sum_{k} d_{k}^{j} \otimes d_{i}^{k} .
$$

This coproduct and the counit (3.1) endow $D$ with a structure of a coassociative coalgebra. Moreover, since this structure is coordinated with the algebraic structure of $D$, this algebra becomes a bi-algebra.

Now, the standard action $d_{i}^{j}\left(n_{k}^{l}\right)=\delta_{i}^{l} \delta_{k}^{j}$ the derivatives $d_{i}^{j}$ on the generators $n_{k}^{l}$ of the algebra $U\left(g l(m)_{h}\right)$ can be extended to the whole algebra $U\left(g l(m)_{h}\right)$ via the above coproduct. In order to prove that the extended action respects the algebraic structure of $U\left(g l(m)_{h}\right)$, it suffices to check that the ideal generated in this algebra by the elements

$$
P N_{1} P N_{1}-N_{1} P N_{1} P-h\left(P N_{1}-N_{1} P\right)
$$

is invariant with respect to the action of the derivatives. The detail is left to the reader.

Remark 5: Note that by using the method of [11] it is possible to recover the permutation relations (the third line of (2.1)) on the base of the coproduct (3.2). However, in a more general case related to the Reflection Equation algebra [7] we have not succeeded in finding the coproduct corresponding to the permutation relations studied in this paper. Thus, the permutation relations are in a sense a more fundamental structure enabling us to de ne an action of the algebra $D$ on that $U\left(g l(m)_{h}\right)$ without any coproduct.

Now, pass to the case $m=2$ and extend the derivatives $\partial_{t}, \ldots, \partial_{z}$ onto the algebra $A_{\hbar}$ (here we deal with the compact form of the algebra $\operatorname{gl}(2)_{h}$ and also we use the generator $\tau$ instead of $\left.t\right)$. First, we exhibit the coproduct $\Delta$ in the generators $\partial_{t}, \partial_{x}, \partial_{y}, \partial_{z}$. Thus, we have

$$
\begin{aligned}
& \Delta\left(\partial_{t}\right)=\partial_{t} \otimes 1+1 \otimes \partial_{t}+\frac{h}{2}\left(\partial_{t} \otimes \partial_{t}-\partial_{x} \otimes \partial_{x}-\partial_{y} \otimes \partial_{y}-\partial_{z} \otimes \partial_{z}\right), \\
& \Delta\left(\partial_{x}\right)=\partial_{x} \otimes 1+1 \otimes \partial_{x}+\frac{h}{2}\left(\partial_{t} \otimes \partial_{x}+\partial_{x} \otimes \partial_{t}+\partial_{y} \otimes \partial_{z}-\partial_{z} \otimes \partial_{y}\right),
\end{aligned}
$$

By applying the cyclic permutations to the latter formula, we get $\Delta(\partial)$ and $\Delta\left(\partial_{z}\right)$.

Now, using formulae (2.16) and (2.15) we can compute the action of the derivatives $\partial_{\tau}=i \partial_{t}$ and $\partial_{x}$ on $f\left(\tau, r_{\hbar}\right)$ :

$$
\begin{array}{ll}
\partial\left(f\left(\tau, \mathrm{r}_{h}\right)\right)=\frac{f\left(\tau+\hbar, \mathrm{r}_{h}+\hbar\right)\left(\mathrm{r}_{h}+\hbar\right)+f\left(\tau+\hbar, \mathrm{r}_{h}-\hbar\right)\left(\mathrm{r}_{h}-\hbar\right)-2 \mathrm{r}_{h} f\left(\tau, \mathrm{r}_{h}\right)}{2 \mathrm{r}_{\hbar} \hbar}, & \text { (3.3) patt } \\
\partial_{x}\left(f\left(\tau, \mathrm{r}_{h}\right)\right)=\frac{x}{\mathrm{r}_{\hbar}} \frac{\left(f\left(\tau+\hbar, \mathrm{r}_{\hbar}+\hbar\right)-f\left(\tau+\hbar, \mathrm{r}_{\hbar}-\hbar\right)\right)}{2 \hbar} . & \text { (3.4) pax }
\end{array}
$$

Similar formulae are valid for $\partial_{y}\left(f\left(\tau, \mathrm{r}_{\hbar}\right)\right) \operatorname{or} \partial_{z}\left(f\left(\tau, \mathrm{r}_{\hbar}\right)\right)$

Extend the action of the derivatives on the algebra $A_{\hbar}$ via the above coproduct. In order to show that the extended derivatives are well defined on this algebra it sufficed to check that they map the ideal generated in this algebra by the elements

$u g\left(\tau, \mathrm{r}_{\hbar}\right)-g\left(\tau, \mathrm{r}_{\hbar}\right) u, u \in\{\tau, x, y, z\}$

to itself. The following proposition entails this property.

Proposition 6: Each element

$\partial_{v}\left(u f\left(\tau, \mathrm{r}_{h}\right)-f\left(\tau, \mathrm{r}_{\hbar}\right) u\right), u, v \in\{\tau, x, y, z\}$

(3.6) that

is a linear combinations of the elements of the form (3.5).

Proof: In fact, it suffices to check (3.6) with $v=x$ and $u=y$. Other relations are trivial or can be obtained by the cyclic permutation of the generators $x, y, z$. Checking (3.6) with $v=x, u=y$ can be done with the help of formula (3.4) or its equivalent form (4.1) below.

Note that any element of the algebra $W\left(U\left(u(2)_{h}\right)\right)$ can be presented as a finite sum of elements $\partial_{z}^{d} \partial_{y}^{c} \partial_{z}^{b} \partial_{t}^{a}, a, b, c, d \in \mathbb{N}$ with coefficients from $U\left(u(2)_{h}\right)$ (we put them on the left hand side). In a similar way we can realize elements of the algebra $W\left(A_{\hbar}\right)$. This realization of any element of the algebra $W\left(U\left(u(2)_{h}\right)\right)$ or $W\left(A_{\hbar}\right)$ is called canonical. A canonical form of a given element $A \in W\left(\mathrm{~A}_{h}\right)$ allows us to assign to $A$ an operator $D_{A}: A_{\hbar} \rightarrow A_{\hbar}$ (for this assignment we use the operator meaning of elements from $D$ discussed above).

Let us denote Diff the vector space of all such differential operators. Thus, we have an isomorphism

$$
\text { Iso : } W\left(A_{\hbar}\right) \rightarrow \text { Diff }
$$

which is a representation of the algebra $\mathrm{W}\left(A_{\hbar}\right)$.

Now we construct a special basis in the algebra $U\left(u(2)_{h}\right)$ useful in the sequel. First, note that $U\left(u(2)_{h}\right)=U(s u(2) h) \otimes \mathbb{R}[t]$. Second, we use the fact that the algebra $U(s u(2) h)$ is free over its center $Z(U(s u(2)$ h) ) (Kostant's theorem, see [D]). Let $V^{k} \subset U\left(s u(2)_{h}\right), k \geq 0$ (we set $\left.V^{0} \equiv \mathbb{R}\right)$, be an irreducible adjoint $U\left(\operatorname{su}(2)_{h}\right)$ submodule such that $b^{k}=(-i x-y)^{k}$ is a highest weight element in its complexification $V_{C}^{k}$. Thus, $V^{k}$ is the submodule of the spin $k, \operatorname{dim} V^{k}=2 k+1$.

Then we have

$$
U\left(s u(2)_{h}\right)=\bigoplus_{k=0}^{\infty}\left(Z\left(U\left(s u(2)_{h}\right)\right) \otimes V^{k}\right) .
$$

As is known, $Z\left(U\left(s u(2)_{h}\right)\right)$ consists of all polynomials in Cas. By fixing a basis $\left\{v_{i}^{k} \leq 1 \leq i \leq 2 k+1\right\}$ in each submodule $V^{k}$ and the basis $\left\{\mathrm{Cas}^{\mathrm{p}}\right\}, \mathrm{p} \geq 0$ in $Z\left(U\left(\operatorname{su}(2)_{h}\right)\right)$, we get a basis $\left\{\mathrm{Cas}^{p} \otimes v_{i}^{k}\right\}$ of the whole algebra $U\left(\operatorname{su}(2)_{h}\right)$.

A similar decomposition hold true for the algebras $U\left(u(2)_{h}\right)$ and $A_{\hbar}$ :

$$
U(u(2) \mathrm{h})=\bigoplus_{k=0}^{\infty}\left(Z\left(U\left(u(2)_{h}\right)\right) \otimes V^{k}\right), \quad{ }_{h}=\bigoplus_{k=0}^{\infty}\left(Z(\mathrm{~A}) \otimes V^{k}\right) .
$$


Consider now a natural action of the rotation group $S O(3)$ on the algebra $W\left(A_{\hbar}\right)$ (and consequently on Diff). This group acts on the "spacial generators" $x, y, z$ of the algebra $W\left(A_{\hbar}\right)$ and on the corresponding derivatives $\partial_{\mathrm{x}}, \partial_{\mathrm{y}}$ and $\partial_{\mathrm{z}}$ in the classical way, but it affects neither the factor $\mathbb{k}\left(\tau, \mathrm{r}_{\hbar}\right)$ nor the derivative $\partial_{T}$.

Definition 7: We call a differential operator $D_{A} \in$ Diff to be invariant if it is the image of an $S O(3)$-invariant element $A \in W\left(A_{h}\right)$ of the algebra $W\left(A_{\hbar}\right)$ under the isomorphism $\mathrm{W}\left(A_{\hbar}\right) \rightarrow$ Diff.

Important examples of the first order invariant operators are

$\bar{\partial}_{t} \quad$ and $\quad Q=x \partial_{x}+y \partial_{y}+z \partial_{z}$.

(3.7) first

Some the second order differential operators to be used below are

$\Delta_{0}=\bar{\partial}_{t}^{2}, \quad \Delta_{1}=\Delta=\partial_{x}^{2}+\partial_{y}^{2}+\partial_{z}^{2}, \quad \Delta_{2}=Q \bar{\partial}_{t}, \quad \Delta_{3}=Q^{2}$. (3.8) second

All operators from the lists (3.7) and (3.8) will be called basic. The operator $\Delta$ is called Laplacian. All polynomials in the basic operators with coefficients from $\mathbb{k}\left(\tau, \mathrm{r}_{\hbar}\right)$ are also invariant operators.

Proposition 8: Let $D: A_{\hbar} \rightarrow A_{\hbar}$ be a basic invariant differential operator. Then it maps the component

$$
v^{k}=\mathbb{k}\left(\tau, \mathrm{r}_{\hbar}\right) \otimes V^{k}
$$

to itself. Moreover, its action on any element $f\left(\tau, \mathrm{r}_{\hbar}\right) v, v \in V^{k}$ alters only the central function $f\left(\tau, \mathrm{r}_{\hbar}\right)$. Thus, we have

$\mathrm{D}\left(f\left(\tau, \mathbf{r}_{\hbar}\right) v\right)=g\left(\tau, \mathbf{r}_{\hbar}\right) v$

for some $g\left(\tau, \mathrm{r}_{\hbar}\right) \in \mathbb{k}\left(\tau, r_{\hbar}\right)$.

Proof: First, observe that the evaluation map

Diff $\otimes A_{\hbar} \rightarrow A_{\hbar}, D \otimes n \mapsto D(n)$

is $S O(3)$-covariant. This entails that if $D$ is an invariant differential operator it commutes with the action of the group $S O(3)$. Therefore, its complexification commutes with the action of the group $\operatorname{SL}(2, \mathbb{C})$.

Now we use the following fact, proved in [GS2].

Lemma 9: The action of the partial derivatives $\partial_{t}, \ldots, \partial_{z}$ onto the elements $b^{k} \in V_{c}^{k}$ is subject to the classical Leibniz rule.

Due to lemma 9 we can conclude that all basic differential operators act on the elements $b^{k}$ by the classical formula. Namely, we have

$$
\begin{aligned}
& \partial_{t}\left(b^{k}\right)=0, \quad Q\left(b^{k}\right)=k b^{k} \\
& \Delta\left(b^{k}\right)=0, \quad \Delta_{0}\left(b^{k}\right)=\left(\frac{2}{h}\right)^{2} b^{k}, \quad \Delta_{2}\left(b^{k}\right)=\frac{2 k}{h} b^{k}, \quad n \Delta_{3}\left(b^{k}\right)=k^{2} b^{k} . n
\end{aligned}
$$

Now, the claim of the proposition follows immediately from the invariancy of the basic operators.

As follows from the above consideration, the restriction of any basic operator to the components $V^{k}$ can be well defined. Note that being restricted to the component $V^{k}$ such an operator acts as a difference operator on the factor $f\left(\tau, r_{\hbar}\right)$ in an element $f\left(\tau, r_{\hbar}\right) v, v \in V^{k}$. The reader is referred to [7] for explicit examples and detail.

Thus, the spectral problem for a basic differential operator $D$

$$
\mathrm{D}(f)=\lambda f, \quad f \in A_{\hbar}
$$

reduces to the spectral problem of a family of its restrictions to the components $V^{k}$

Definition 10: The restriction of a basic operator $D$ on the component $V^{0}=K\left(\tau, \mathrm{r}_{h}\right)$ is called the radial part of the operator $D$ and is denoted $D_{r a d}$
As follows from [GS2], the radial part of the Laplacian $\Delta$ has the form:

$$
\begin{array}{r}
\Delta_{\text {rad }}\left(f\left(\tau, \mathrm{r}_{h}\right)\right)=\frac{f\left(\tau+\hbar, \mathrm{r}_{\hbar}+2 \hbar\right)+f\left(\tau+\hbar, \mathrm{r}_{\hbar}-2 \hbar\right)-2 f\left(\tau+\hbar, \mathrm{r}_{\hbar}\right)}{4 \hbar \hbar^{2}} \\
+\frac{1}{r_{\hbar}} \frac{f\left(\tau+\hbar, \mathrm{r}_{h}+2 \hbar\right)-f\left(\tau+\hbar, \mathrm{r}_{\hbar}-2 \hbar\right)}{2 \hbar}
\end{array}
$$

Another important example is the radial part of the operator $Q$

$$
Q_{r a d}\left(f\left(\tau, \mathrm{r}_{\hbar}\right)\right)=\frac{\left(\mathrm{r}_{\hbar}^{2}-\hbar^{2}\right)}{r_{\hbar}} \frac{\left(f\left(\tau+\hbar, \mathrm{r}_{\hbar}+\hbar\right)-f\left(\tau+\hbar, \mathrm{r}_{\hbar}-\hbar\right)\right)}{2 \hbar} .
$$

This result immediately follows from (3.4) and its $y$ - and $z$-analogs.

\section{Quantum Radial Derivative and its Applications}

Let us introduce the operator $\partial_{r_{n}}: \mathbb{k}\left(\tau, r_{\hbar}\right) \rightarrow \mathbb{k}\left(\tau, r_{\hbar}\right)$ by the following rule

$$
\partial_{r h} f\left(\tau, \mathrm{r}_{\hbar}\right)=\frac{f\left(\tau+\hbar, \mathrm{r}_{\mathrm{h}}+\hbar\right)-f\left(\tau+\hbar, \mathrm{r}_{h}-\hbar\right)}{2 \hbar}, \quad \forall f\left(\tau, r_{\hbar}\right) \in K\left(\tau, r_{\hbar}\right) .
$$

This operator is well defined on the component $\mathbb{k}\left(\tau, \mathbf{r}_{\hbar}\right)$ and we call it the quantum radial derivative. It is a difference operator which turns into the usual derivative in $r$ as $\hbar \rightarrow 0$ :

$$
\lim _{\hbar \rightarrow 0} \partial_{r_{h}} f\left(\tau, \mathrm{r}_{\hbar}\right)=\frac{\partial f(\tau, r)}{\partial r} .
$$

With this notation we rewrite formula (3.4) in the form:

$$
\partial_{x}\left(f\left(\tau, \mathrm{r}_{\hbar}\right)\right)=\frac{x}{r_{\hbar}} \partial_{\mathrm{r}_{h}}\left(f\left(\tau, \mathrm{r}_{\hbar}\right)\right) . \quad \text { (4.1) } \mathrm{X} \text { case }
$$

This formula is similar to the classical one but with another meaning of the derivative. Formula (3.11) can also be rewritten in terms of the new derivative:

$$
Q_{\text {rad }}\left(f\left(\tau, \mathrm{r}_{\hbar}\right)\right)=\frac{\left(\mathrm{r}_{\hbar}^{2}-\hbar^{2}\right)}{r_{\hbar}} \partial r_{\hbar}\left(f\left(\tau, \mathrm{r}_{\hbar}\right)\right) .
$$

Since the function $f\left(\tau, r_{\hbar}\right)$ is an arbitrary element of $\mathbb{k}\left(\tau, \mathrm{r}_{\hbar}\right)$ we have an operator equality on the algebra $\mathbb{k}\left(\tau, \mathrm{r}_{\hbar}\right)$

$$
\partial r_{\hbar}=\frac{r_{\hbar}}{r_{\hbar}^{2}-\hbar^{2}} Q \text {. }
$$

We use this relation as the definition of an extension of the quantum radial derivative $\partial_{r h}$ on the whole algebra $A_{h}$. Note that this definition differs from that from [7] by the factor at the operator $Q$.

As the next step we express the operator $\Delta_{r a d}$ in terms of the quantum radial derivative. A straightforward calculation on the base of relation (3.11) leads to the result:

$\Delta_{\text {rad }}\left(f\left(\tau, \mathrm{r}_{\hbar}\right)\right)=\frac{1}{r_{\hbar}} \partial_{\mathrm{r}_{\hbar}}^{2}\left(\mathrm{r}_{\hbar} f\left(\tau, \mathrm{r}_{\hbar}\right)\right)$.

Remark 11: Formula (4.3) is analogous to the classical one

$$
\Delta_{r a d}(f(t, r))=\frac{1}{r} \partial_{r}^{2}(r f(t, r))=\partial_{r}^{2}(f(t, r))+\frac{2}{r} \partial_{r}(f(t, r)) \text { (4.4) r Lap }
$$

and turns into this as $\hbar \rightarrow 0$. Actually, formula (4.3) has motivated our definition of the quantum radial derivative.

In contrast with the classical case, the Laplace operator $\Delta$ affects the variable $\tau$, though it does not explicitly contain the derivative $\partial_{T}$. This effect disappears as $\hbar \rightarrow 0$.

Our next aim is to solve the equation

$$
\Delta\left(f\left(r_{\hbar}\right)\right)=\Delta_{\text {rad }}\left(f\left(r_{\hbar}\right)\right)=0
$$

for a $\tau$-independent function $f\left(\mathrm{r}_{\hbar}\right) \in Z\left(\mathrm{~A}_{\hbar}\right)$. In the classical case 
$(\hbar=0)$ the vector space of solutions of the equation $\Delta_{r a d}(f(r))=0$ in the region $r>0$ consists of all linear combinations of two functions $f_{1}(r)=1$ and $f_{2}(r)=r^{-1}$.

In the quantum case as follows from expression (4.3) the functions $f_{1}\left(r_{\hbar}\right)=1$ and $f_{2}\left(r_{\hbar}\right)=r_{\hbar}^{-1}$ are solutions of the equation (4.5). Moreover, all linear combinations $C_{1}\left(r_{\hbar}\right) f_{1}+C_{2}\left(\mathrm{r}_{\hbar}\right) f_{2}$ are also solutions provided that the coefficients $C_{i}\left(r_{\hbar}\right), i=1,2$, are $2 \hbar$ periodical functions in $r_{\hbar}$. Remark 12: In the classical case the function $\frac{1}{r}=\frac{1}{\sqrt{x^{2}+y^{2}+z^{2}}}$
satisfies the equation

$$
\Delta\left(\frac{1}{r}\right)=-4 \pi \delta(\mathbf{x})
$$

on the whole space $\mathbb{R}^{3}$. Here, $x=(x, y, z)$ and $\delta(\mathrm{x}-\mathrm{a})$ stands for the $\delta$-function located at the point $\mathrm{a}=\left(a_{1}, a_{2}, a_{3}\right) \in \mathbb{R}^{3}$. In section 6 we shall discuss a quantum version of this equation.

Note that the operator (4.3) is well defined on a lattice

$$
\left(\mathrm{r}_{h}, \tau\right) \in\{a+2 k \hbar, b+l \hbar, k, l \in \mathbb{Z}\},
$$

where $a$ and $b$ are some fixed real numbers. Thus, we can consider the restriction of this operator to the lattice (4.6). A similar restriction is possible for any operator depending only on the quantities $\tau$ and $r_{\hbar}$ and the derivatives $\partial_{T}$ and $\partial_{r h}$. In this sense we speak about the discretization of a dynamical odel escribed by such an operator.

This discretization reduces the freedom for coefficients in the space of solutions of the equation $\Delta\left(f\left(\tau, r_{\hbar}\right)\right)=0$. Thus, the aforementioned coefficients $C_{i}\left(r_{\hbar}\right)$ entering the general solution become constant on each of the two sublattices $\{a+2 k \hbar, b+l \hbar, k, l \in \mathbb{Z}\}$ and

$$
\{a+\hbar+2 k \hbar, b+l \hbar, k, l \in \mathbb{Z}\}
$$

Let us assume the algebra $U(g l(2))$ to be represented in a Verma module $M_{\Lambda}$ of the highest weight $\Lambda=\left(\Lambda_{1}, \Lambda_{2}\right), \Lambda_{1} \geq \Lambda_{2}$ (for the definition we refer the reader to the book [12]. The module $M_{\Lambda}$ has a finite dimensional $U(g l(2))$ submodule if $\Lambda_{1}-\Lambda_{2}=n$ is a non-negative integer. On passing to the algebra $U(g l(2))$ we get (after rescaling of the generators $x \rightarrow x / h$ etc.) the corresponding representation $\rho_{\Lambda}: U\left(u(2)_{h}\right) \rightarrow\left(M_{\Lambda}\right)$, which can also be factorized up to a finite dimensional representation provided $\Lambda_{1}-\Lambda_{2}=n$. (Note that $\frac{n}{2}$ is the spin of this finite dimensional representation).

It is known that the image of any central element $u \in Z\left(U\left(u(2)_{h}\right)\right)$ under the representation $\rho_{\Lambda}$ is a scalar operator (it will be identified with its eigenvalue). In particular, we have

$$
\rho \Lambda(\text { Cas })=\rho \Lambda=\left(\Lambda_{1}-\Lambda_{2}\right)\left(\Lambda_{1}-\Lambda_{2}+2\right) \hbar .
$$

This enables us to compute the corresponding value of the quantum radius $r_{\hbar}$. Namely, we have

$$
\rho_{\Lambda}\left(\mathrm{r}_{h}\right)=\sqrt{\left(\Lambda_{1}-\Lambda_{2}+1\right)^{2} \hbar^{2}}=\left(\Lambda_{1}-\Lambda_{2}+1\right) \hbar .
$$

The sign here is motivated by our wish to have a positive quantum radius (at $\hbar>0$ ) on finite dimensional modules. Thus, on the finite dimensional module of the spin $\frac{n}{2}$ the quantum radius takes the values

$$
r h=(n+1) \mathrm{h}, \quad n \in \mathbb{Z}_{+} .
$$

So, the operator $\Delta_{\text {rad }}$ admits a discretization well defined on a family of the Verma modules, some of them can be factorized to finite dimensional ones. This is also true for the isotypic components $V^{\mathrm{k}}$ (3.9) with $k \neq 0$.
We complete this section with the following observation. Let

$$
\alpha: \operatorname{sym}(u(2)) \rightarrow U(u(2) \mathrm{h})
$$

be an $S U(2)$-covariant quantizing map [13]. Then any operator

$$
D: \operatorname{sym}(u(2)) \rightarrow \operatorname{Sym}(u(2))
$$

can be pulled forward to

$$
D_{\alpha}: U\left(u(2)_{h}\right) \rightarrow U\left(u(2)_{h}\right), \quad D_{\alpha}=\alpha D \alpha^{-1} .
$$

However, the image of a usual partial derivative in the algebra $\operatorname{Sym}(u(2))$ with respect to the map (4.8) does not coincide with the quantum partial derivatives considered in the present paper. Nevertheless, the images of the infinitesimal rotations in $\operatorname{Sym}(u(2))$ remain to be infinitesimal rotations in $U\left(u(2)_{h}\right)$.

As for the boosts, their analogs are not naturally defined on the algebra $U\left(u(2)_{h}\right)$. Let us introduce the infinitesimal "radial boost" in the classical case $(\hbar=0)$

$$
t \partial_{r}+r \partial_{t}=t \frac{Q}{r}+r \partial_{t} .
$$

The corresponding one parameter group $\exp \left(v\left(t \partial_{r}+r \partial_{t}\right)\right)$ preserves the Lorentz interval $s=\sqrt{t^{2}-\left(x^{2}+y^{2}+z^{2}\right)}=\sqrt{t^{2}-r^{2}} \quad$ since

$\left(t \partial_{r}+r \partial_{t}\right)(s)=0$.

We define the quantum analog of the "infinitesimal radial boost" as $\tau \partial_{r_{h}}+r_{h} \partial_{\tau}$

and the "quantum Lorentz interval" squared as $\tau^{2}-r_{\hbar}^{2}$. However, now the action

$$
\left(\tau \partial_{r_{\hbar}}+r_{h} \partial_{\tau}\right)\left(\tau^{2}-r_{\hbar}^{2}\right)=\tau\left(-2 r_{\hbar}\right)+r_{\hbar}(2 \tau-2 \hbar)=-2 \hbar r_{\hbar}
$$

is proportional to $\mathrm{h}$ and disappears only as $\hbar \rightarrow 0$.

\section{Klein-Gordon and Schrodinger Equations on $\mathrm{U}\left(\mathrm{u}(2)_{\mathrm{h}}\right)$}

In this section we consider the NC (quantum) versions of two dynamical models playing an important role in physics. Namely, we are dealing with the Klein-Gordon equation (as an example of a relativistic model) and the Schrodinger equation with the hydrogen atom potential (as an example of a non-relativistic model). For the former equation we exhibit an analog of the plane wave solution under an assumption on momenta parameterizing the solution. With this assumption we get a deformed version of the relativistic dispersion relation. For the latter equation we are looking for a value of the ground state energy.

\section{The Klein-Gordon equation}

The Klein-Gordon equation describes evolution of a free massive scalar field. It is the second order partial differential equation of the form (we assume the speed of light and the Planck constant to be equal to the unity)

$$
\left(\partial_{t}^{2}-\partial_{x}^{2}-\partial_{y}^{2}-\partial_{z}^{2}+m^{2}\right)(\phi(t, x, y, z))=0 .
$$

Here $\mathrm{m}$ is a real constant (the mass of the eld). This equation possesses the plane wave solutions

$$
\phi(t, x, y, z)=\exp i\left(E t-p_{x} x-p_{y} y-p_{z} z\right)
$$

where the energy $\mathrm{E}$ and the momenta $\left(p_{x}, p_{y}, p_{z}\right)$ are subject to the relativistic dispersion relation

$$
E^{2}=p_{x}^{2}+p_{y}^{2}+p_{z}^{2}+m^{2} .
$$


Let us pass to a NC version of the Klein-Gordon equation. Since the generators $x, y, z$ do not explicitly enter equation (5.1), this passage is straightforward we have to assign a new meaning to the derivatives expressed in the relations (2.3).

Note that in the NC case we meet an additional difficulty. The symbol $\phi(\tau, x, y, z)$ is not well defined now, since we have to explicitly fix some ordering of the NC variables $x, y$ and $z$. To avoid this technical difficulty, we consider a solution depending on the central element $\tau$ and the only spacial variable, say $x$. Otherwise stated, we assume the momentum to be of the form $p=\left(p_{x}, 0,0\right)$.

Remark 13: This simplification enables us to compute the action of the Klein-Gordon operator on a plane wave (5.2). Note that since the element $t, x, y, z$ belong to the algebra $U\left(u(2)_{h}\right)$, this plane wave can be treated as an element of the group $U(2)$ (at least for the small enough coefficients $E$ and $p$ ) presented in the coordinates of the first kind. The computation becomes easier if we present elements of this group in the coordinates of the second kind. This means that the function $\phi$ has the form $\exp (i E t) \exp \left(-i p_{x} x\right) \exp \left(-i p_{y} y\right) \exp \left(-i p_{z} z\right)$. An example of computation with such a function is given in [6].

First of all, we note, that similarly to the classical case

$$
\left(\partial_{y}^{2}+\partial_{z}^{2}\right)(\phi(\tau, x))=0 \text {. }
$$

This immediately follows from formula (7.1) of Appendix. So, our equation becomes

$$
\left(\partial_{\tau}^{2}-\partial_{x}^{2}+m^{2}\right)(\phi(\tau, x))=0 .
$$

We look for solutions of this equation in the form

$$
\phi(\tau, x)=f_{E}(\tau) g_{p}(x)
$$

where $E$ and $p=p_{x}$ are numeric parameters of the solution (analogs of the energy and momentum of the commutative case). Our goal is to find the explicit form of the functions $f_{E}(\tau)$ and $g_{p}(x)$ and the dispersion relation, connecting $E$ and $p$.

Let us demand the function $f_{E}(\tau)$ to be an eigenfunction of the derivative $\partial_{\tau}$ with the eigenvalue $E$. The action of the derivative $\partial_{\tau}$ on a function in $\tau$ can be extracted from (2.14). So, we have

$$
\partial_{\tau}\left(f_{E}(\tau)\right)=\frac{f_{E}(\tau+\hbar)-f_{E}(\tau)}{\hbar}=E f_{E}(\tau) .
$$

The solution of this difference equation reads:

$$
f_{E}(\tau)=\xi(E)^{\tau / \hbar} \text {, where } \xi(E)=1+\hbar E .
$$

Note that the function $f_{E}(\tau)$ can be presented in the form $f_{E}(\tau)=\exp (\alpha(E) \tau)$, where $\alpha(\mathrm{E})=\frac{\ln (1+\hbar \mathrm{E})}{\hbar}$. In the classical limit $\hbar \rightarrow 0$ the function $\alpha(\mathrm{E})$ turns into $E$.

In a similar way, the function $g_{p}(x)$ is taken to be an eigenfunction of the operator $\partial_{x}$ with the eigenvalue $p$ :

$$
\partial_{x}\left(g_{p}(x)\right)=\frac{g_{p}(x+\hbar)-g_{p}(x-\hbar)}{2 \hbar}=p g_{p}(x) .
$$

We take the solution of this equation in the form

$$
g_{p}(x)=\eta(p)^{x / \hbar}, \text { where } \eta(p)=\hbar p+\sqrt{1+(\hbar p)^{2}} .
$$

In the classical limit we get $\lim _{h \rightarrow 0} g_{p}(x)=e^{x p}$.

Let us now turn to solving equation (5.3) with the above substitution $\phi(\tau, x)=f_{E}(\tau) g_{p}(x)$. First of all, permuting $\partial_{\tau}^{2}$ and $f_{E}(\tau)$ we have: $\partial_{\tau}^{2} f_{E}(\tau)=E^{2} f_{E}(\tau)+2 E f_{E}(\tau+\hbar) \partial_{\tau}+f_{E}(\tau+2 \hbar) \partial_{\tau}^{2}=f_{E}(\tau)\left(E^{2}+2 E \xi \partial_{\tau}+\xi^{2} \partial_{\tau}^{2}\right)$.

Also (see Appendix for detail)

$$
\partial_{\tau}\left(g_{p}(x)\right)=g_{p}(x) \frac{(\eta-1)^{2}}{2 \hbar \eta}, \quad \partial_{\tau}^{2}\left(g_{p}(x)\right)=g_{p}(x) \frac{(\eta-1)^{4}}{(2 \hbar \eta)^{2}} .
$$

In the above formulae we omit the arguments $\mathrm{E}$ and $\mathrm{p}$ in the notations $\xi(E)$ and $\eta(p)$ respectively. All these formulae follow from the permutation relations of the partial derivatives in $\tau$, presented in Appendix, and the particular form of the functions $f_{E}(\tau)$ and $g_{p}(x)$. This allows us to calculate the term $\partial_{\tau}^{2}(\phi(\tau, x))$ in (5.3).

In order to find the value of the second term $\partial_{x}^{2}(\phi(\tau, x))$, we use the relation

$$
\partial_{x}^{2}\left(f_{E}(\tau) g_{p}(x)\right)=f_{E}(\tau+2 \hbar) \partial_{x}^{2}\left(g_{p}(x)\right)=p^{2} \xi^{2} f_{E}(\tau) g_{p}(x) .
$$

Finally, we come to the equation:

$$
f_{E}(\tau) g_{p}(x)\left[\left(\xi \frac{(\eta-1)^{2}}{2 \hbar \eta}+E\right)^{2}-\xi^{2} p^{2}+m^{2}\right]=0,
$$

which, in turn, leads to the dispersion relation, connecting the parameters $E$ and $p$

$$
\left(\xi(E) \frac{(\eta(p)-1)^{2}}{2 \hbar \eta(p)}+E\right)^{2}-\xi(E)^{2} p^{2}+m^{2}=0 .
$$

On taking into account the explicit form of $\xi(E)$ and $\eta(p)$, we simplify the dispersion relation to the form

$$
E^{2}-2 p^{2} \frac{1+\hbar E}{1+\sqrt{1+(\hbar p)^{2}}}+m^{2}=0 .
$$

On solving the equation in $E$ we find

$$
E_{ \pm}= \pm \sqrt{p^{2}-m^{2}}+\frac{\hbar p^{2}}{1+\sqrt{1+(\hbar p)^{2}}} .
$$

This is the final form of the "quantum dispersion relation" under the above assumption $p=p_{x}, p_{y}=p_{z}=0$.

\section{The Schrodinger type equation}

Now, let us consider a differential equation of the Schrodinger type

$$
\left(a \partial_{\tau}+b \Delta+\frac{q}{r_{h}}\right)(\psi)=0
$$

where $r_{\hbar}$ is a quantum radius and $a, b, q$ are some numeric constants. It is convenient to look for $\psi$ in the form

$$
\psi=\psi^{(k)}\left(\tau, \mathrm{r}_{\mathrm{h}}\right) v=f_{E}(\tau) \phi\left(\mathrm{r}_{\mathrm{h}}\right) v,
$$

where $v \in V^{k}$ and $\psi^{(k)}\left(\tau, r_{h}\right) \in Z\left(A_{h}\right)$ and $f_{E}(\tau)$ is defined by formula (5.4).

Below we are dealing with the particular case $k=0$ of this problem. Using the properties of $f_{E}(\tau)$ we get the equation on $\phi\left(r_{h}\right)$ :

$$
\begin{aligned}
a E \phi\left(\mathrm{r}_{\hbar}\right)+ & a \xi(E) \frac{\phi\left(\mathrm{r}_{\hbar}+\hbar\right)\left(\mathrm{r}_{\hbar}+\hbar\right)+\phi\left(\mathrm{r}_{h}-\hbar\right)\left(\mathrm{r}_{\hbar}-\hbar\right)-2 r_{\hbar} \phi\left(\mathrm{r}_{\hbar}\right)}{2 \hbar r_{\hbar}} \\
& +b \xi(E)^{2}\left[\frac{\phi\left(\mathrm{r}_{\hbar}+2 \hbar\right)-\phi\left(\mathrm{r}_{\hbar}-2 \hbar\right)}{2 \hbar r_{\hbar}}+\frac{\phi\left(\mathrm{r}_{\hbar}+2 \hbar\right)+\phi\left(\mathrm{r}_{\hbar}-2 \hbar\right)-2 \phi\left(\mathrm{r}_{\hbar}\right)}{4 \hbar^{2}}\right] \\
& +\frac{q}{r_{\hbar}} \phi\left(\mathrm{r}_{\hbar}\right)=0,
\end{aligned}
$$

where $\xi(E)$ is defined in (5.4).

Now, we are interested in question: is there a ground state of the 
$\mathrm{m} \phi\left(\mathrm{r}_{\hbar}\right)=e^{-\sigma r_{h}}$ (similarly to the commutative case) Substituting this exponent into the equation, we get

$$
\begin{aligned}
a E r_{h}+\frac{a \xi(E)}{2 \hbar}\left(\mathrm{r}_{\mathrm{h}}\left(e^{\sigma \hbar}+e^{-\sigma \hbar}-2\right)-\hbar\left(e^{\sigma \hbar}-e^{-\sigma \hbar}\right)\right) & \\
& +\frac{b \xi(E)^{2}}{4 \hbar^{2}}\left[r_{h}\left(e^{2 \sigma \hbar}+e^{-2 \sigma \hbar}-2\right)-2 \hbar\left(e^{2 \sigma \hbar}-e^{-2 \sigma \hbar}\right)\right]+q=0 .
\end{aligned}
$$

Collecting separately the terms with and those without it, we get a system of equations to determine $\sigma$ and $E$ :

$$
\left\{\begin{array}{l}
a E+2 \frac{a \xi(E)}{\hbar} \sinh ^{2}(\sigma \hbar / 2)+\frac{b \xi^{2}(E)}{\hbar^{2}} \sinh ^{2}(\sigma \hbar)=0 \\
\xi(E) \sinh (\sigma \hbar)+\frac{b \xi^{2}(E)}{\hbar} \sinh (2 \sigma \hbar)-q=0
\end{array}\right.
$$

Or

$$
\left\{\begin{array}{l}
\xi(E)=2 \frac{\cosh (\sigma \hbar)-\sinh (\sigma \hbar) q /(2 a)}{1+\cosh ^{2}(\sigma \hbar)} \\
\xi^{2}(E)=\frac{a \hbar}{b} \frac{1-\xi(E) \cosh (\sigma \hbar))}{\sinh ^{2}(\sigma \hbar)}
\end{array} .\right.
$$

This system reduces to the equation on $y=\tanh (\sigma \hbar)$ :

$\left(\omega / 2-2 \rho^{2}\right) y^{3}+\rho(4-\omega) y^{2}-(\omega+2) y+2 \rho \omega=0$,

(5.7) eq-tha

where

$$
\rho=\frac{q}{2 a}, \quad \omega=\frac{a \hbar}{b} .
$$

In the commutative limit $\hbar \rightarrow 0$ the above equation gives in the leading order in $\hbar$

$$
\sigma_{0}=\frac{q}{2 b}
$$

while the leading order of the ground state energy can be extracted from the first line of the system (5.6)

$$
E_{0}=-\frac{q}{2 a} \sigma_{0}=-\frac{q^{2}}{4 a b} .
$$

These limit values coincide with the corresponding solution of the differential equation

$$
\left(a E+b\left(\frac{2}{r} \partial_{r}+\partial_{r}^{2}\right)+\frac{q}{r}\right)(\phi(r))=0,
$$

which is the commutative limit of (5.5) with zero value of the orbital momenta. Below, we compute the first order corrections in $\hbar$ to the values $E_{0}$ and $\sigma_{0}$. We do not know any "physical meaning" of other solutions of the equation (5.7).

The first order corrections in $\hbar$ to the classical results (5.8) and (5.9) can be found from the system (5.6). We present the result of calculations by passing to the physical meaning of the parameters $a, b, q$ entering the equation (5.5). More Precisely, we set $a=h_{p l} c, b=h_{p l}^{2} / 2 m, q=e^{2}$, where $h_{p l}$ is the Planck constant, $c$ is the speed of light, $m$ is the electron mass, $e^{2}$ is the electron charge squared. Besides, we must renormalize the energy parameter $E \rightarrow E /\left(h_{p l} c\right)$. It is convenient to introduce the dimensionles fine structure constant $\alpha$ and the electron De Broglie wave length $\lambda_{B}$ by the relations

$$
\alpha=\frac{e^{2}}{h_{p l} c}, \lambda_{B}=\frac{h_{p l}}{m c} \text {. }
$$

With this constant our answer has the following simple form:

$$
\begin{aligned}
& E=-m c^{2} \frac{\alpha^{2}}{2}\left(1-\frac{\hbar}{4 \lambda_{B}}\left(1-\frac{\alpha^{2}}{2}\right)\right)+O\left(\hbar^{2}\right) \\
& \sigma=\frac{\alpha}{2 \lambda_{B}}\left(1-\frac{\hbar}{\lambda_{B}}\left(1-\alpha^{2}\right)\right)+O\left(\hbar^{2}\right) .
\end{aligned}
$$

The above relations show that noncommutativity of the space-time leads to increasing of the ground state energy as well as to increasing of an average size of the hydrogen atom which is roughly proportional to $\sigma^{-1}$ (we assume $\hbar$ to be positive).

As for the eigen function $f_{E}(\tau) e^{-\sigma r_{h}}$, the first order correction to it can be easily obtained with the use of (5.10) and (5.11) and is left for the reader.

\section{Concluding Remarks, Discussion}

1. First, let us go back to the remark 12. The relation exhibited there means that for any sufficiently smooth compactly supported function $f(x, y, z)$ defined on $\mathbb{R}^{3}$ we have

$$
\int_{\mathbb{R}^{3}} \Delta\left(\frac{1}{r}\right) f(x, y, z) d x d y d z=-4 \pi f(0),
$$

where the above integral is treated in the sense of the principal value.

A proof of (6.1) is usually based on the Green identities. We slightly modify this proof in order to adapt it to the quantum case.

Since we consider the function $\frac{1}{r}$ as a distribution, the integral in the left hand side of (6.1) is by definition

$$
\lim _{\varepsilon \rightarrow 0, R \rightarrow \infty} \int_{V(R, \varepsilon)} \frac{1}{r} \Delta(f(x, y, z)) d x d y d z,
$$

where $V(R, \varepsilon)=B(0, R) \backslash B(0, \varepsilon)$ and $B(0, a)$ is the ball with the center at 0 and the radius $a \geq 0$.

Note that it suffices to assume the function $f$ to be dependent on the radius $r$ only. Otherwise, it can be replaced by

$$
g(r)=\frac{1}{4 \pi} \int f(x, y, z) d \Omega
$$

Where $d \Omega$ is the surface 2 -form of the unit sphere. Note that $\mathrm{g}(0)=f(0,0,0)$.

Then, in virtue of (4.4), the integral (6.2) can be written as

$$
4 \pi \int_{\varepsilon}^{R} \frac{1}{r}\left(\frac{1}{r} \partial_{r}^{2}(r f(r))\right) r^{2} d r=4 \pi\left(f(R)+R f^{\prime}(R)-f(\varepsilon)-\varepsilon f^{\prime}(\varepsilon)\right) .
$$

On passing to the limits $R \rightarrow \infty, \varepsilon \rightarrow 0$ and assuming the function $\mathrm{f}$ to be continuously differentiable at $(0,0,0)$ we get the claim.

Heuristically, we can reproduce the computation of (6.2) in the quantum case (using of course (4.3) instead of (4.4)) and get the same result under the following assumptions (actually, definitions)

$$
d x d y d z=d \Omega r_{\hbar} d r_{\hbar} \text { and } \int_{a}^{b} \partial_{r_{h}}\left(f\left(r_{\hbar}\right)\right) d r_{\hbar}=f(b)-f(a) .
$$

Thus, the notion of the integral (including that "in spherical coordinates") can be adapted to the derivative $\partial_{r_{n}}$ in the classical manner. We plan to consider a multidimensional analog of this formula related to the algebra $U\left(\mathrm{gl}(\mathrm{m})_{h}\right)$ in the subsequent publications.

2. Note that we have also considered another basic physical model, namely, 3-dimensional harmonic oscillator. Compared with the hydrogen atom model, the harmonic oscillator is more difficult to deal 
with. The main difficulty here is in the asymptotic of the wave function of the commutative case $\phi(r) \sim e^{-\sigma r^{2}}$. The direct substitution of the non-commutative analog $e^{-\sigma r_{h}^{2}}$ into the corresponding equation leads to an equation which we cannot solve. Apparently, one should find here some another function in $r_{\hbar}$ (with the commutative limit $e^{-\sigma r_{h}^{2}}$ ) which would be more adequate to the problem in the NC case. Another possible way out is a modification of the harmonic oscillator potential (with recovering its classical form at the commutative limit $\hbar \rightarrow 0$ ) in such a way that the exponent $\mathrm{e}^{\mathrm{r} \sim 2}$ would be an eigen function of the modified Hamiltonian.

3. The renormalization of the time variable leads to a change of the step of the corresponding lattice. We do not know what normalization of the time variable and the corresponding step could be "physically motivated".

4. Now, let us compare once more our approach to quantization of dynamical models with that arising from deformation quantization. As we noticed in Introduction, the models introduced in the frameworks of the latter approach make use of the classical derivatives. Only, the usual commutative product of functions on a given Poisson variety is replaced by the corresponding $\star$-product. Note that sometimes such a $\star$-product is introduced via a map (4.7). For instance, this map can be constructed with the help of isotypic components as done in [13]. In this case it can be naturally extended to the completion similar to that .

Then the operator describing a given dynamical model can be pulled forward to the quantum algebra by means of this map (see formula (4.8)). However, this way of quantizing dynamical models is equivalent to the previous one and does not give rise to partial derivatives which could be difference operators. Consider the operator $\partial_{r_{h}}$ defined in this way on the center of the algebra $A_{\hbar}$ (see [GS1]). Since this operator is the image of the derivative $\partial_{r_{n}}$ under the map $f(r) \rightarrow f\left(r_{\hbar}\right)$, it is also

Citation: Gurevich D, Saponov P (2015) Noncommutative Geometry and Dynamical Models on $U(u(2))$ Background. J Generalized Lie Theory Appl 9 : 215. doi:10.4172/1736-4337.1000215 a differential operator. By contrast, the operator $\partial_{r_{n}}$ constructed in the current paper is a difference operator.

\section{Acknowledgement}

This study (research grant No 14-01-0173) was supported by The National Research University Higher School of Economics Academic Fund Program in 2014/2015.

\section{References}

1. Loday JL (1998) Cyclic Homology. Springer, Verlag Berlin, Heidelberg, New York.

2. Tamarkin D, Tsygan B (2001) Cyclic formality and index theorems. Lett Math Phys 56: 85-97.

3. Ginzburg V (2005) Lectures on Noncommutative Geometry.

4. Van Den Bergh M (1998) A Relation between Hochschild Homology and Cohomology for Goren-stein Rings. Proc Amer Math Soc 126: 1345-1348.

5. Landi G (1997) An introduction to Noncommutative Spaces and their Geometries. Lecture Notes in Physics. Springer-verlag, Berlin.

6. Gurevich D, Pyatov P, Saponov P (2012) Braided Weyl algebras and differentia calculus on $\mathrm{U}(\mathrm{u}(2))$. J Geom Phys 62: 1175-1188.

7. Gurevich D, Saponov P (2013) Braided algebras and their applications to Noncommutative Geometry. Advances in Applied Mathematics 51: 228-253.

8. Kupriyanov V (2013) Quantum mechanics with coordinate dependent noncommutativity. J Math Phys.

9. Kupriyanov V (2013) A hydrogen atom on curved noncommutative space. J Phys A Math Theor.

10. Meljanac S, Skoda Z (2007) Leibniz rules for enveloping algebras in symmetric ordering.

11. Gurevich D, Pyatov P, Saponov P (2011) Braided differential operators on quantum algebras. J Geom Phys 61: 1485-1501.

12. Molev A, Nazarov M, Olshanski G (1994) Yangians and classical Lie algebras Mathematical Surveys and Mono-graphs.

13. Gurevich D, Saponov P (2010) Wave operators on quantum algebras via noncanonical quantization. Acta Applicandae Mathematicae 109: 19-38.
Submit your next manuscript and get advantages of OMICS Group submissions

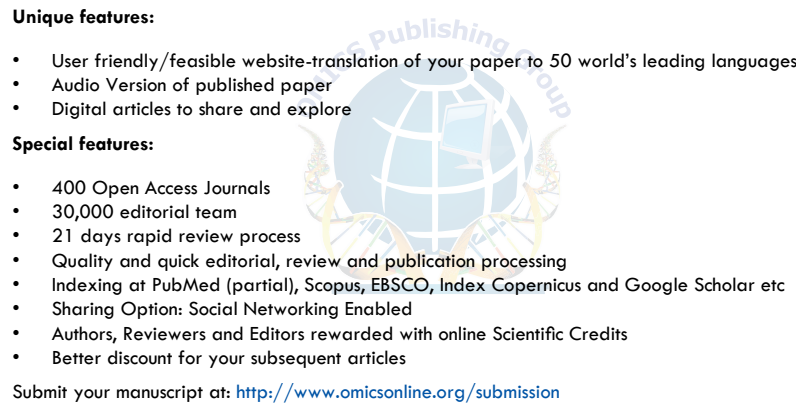

